\title{
Dynamics and variability of winds from single Wolf-Rayet stars
}

\author{
Stanley P. Owocki \\ Bartol Research Institute, University of Delaware, \\ Newark, DE 19350, USA \\ Kenneth G. Gayley \\ Department of Physics and Astronomy, University of Iowa, \\ Iowa City, IA 52245, USA
}

\begin{abstract}
We review the dynamics of winds from single Wolf-Rayet stars, with emphasis on the following specific points:

(a) The classical "momentum problem" (to explain the large inferred ratio of wind to radiative momentum, $\left.\eta \equiv \dot{M} v_{\infty} /(L / c) \gg 1\right)$ is in principle readily solved through multiple scattering of radiation by an opacity that is sufficiently "gray" in its spectral distribution. In this case, one simply obtains $\eta \simeq \tau$, where $\tau$ is the wind optical depth;

(b) Lines with a Poisson spectral distribution yield an "effectively gray" cumulative opacity, with multi-line scattering occuring when the velocity separation between thick lines $\Delta v$ is less than the wind terminal speed $v_{\infty}$. In this case, one obtains $\eta \simeq v_{\infty} / \Delta v$;

(c) However, realistic line lists are not gray, and leakage through gaps in the line spectral distribution tends to limit the effective scattering to $\eta \lesssim 1$;

(d) In WR winds, ionization stratification helps spread line-bunches and so fill in gaps, allowing for more effective global trapping of radiation, and thus $\eta>1$;

(e) However, photon thermalization can reduce the local effectiveness of linedriving near the stellar core, making it difficult for radiation alone to initiate the wind;

(f) The relative complexity of WR wind initiation may be associated with the extensive turbulent structure inferred from observed variabililty in WR wind emission lines;

and $(g)$ Overall, the understanding of WR winds is perhaps best viewed as an "opacity problem", i.e., identifying the enhanced opacity that can adequately block the radiation flux throughout the wind, and thus drive a WR mass loss that is much greater than from OB stars of comparable luminosity.
\end{abstract}

\section{Introduction}

One of the great, longstanding challenges in the study of Wolf-Rayet stars has been to understand the underlying causes and dynamics of their exceptionally strong stellar winds. Traditionally this challenge has been framed as a "momentum problem", focussed on how a radiatively driven wind model could reproduce the very high wind "performance number", $\eta \equiv \dot{M} v_{\infty} /(L / c)$, which represents the ratio of the radial momentum in the stellar wind, $\dot{M} v_{\infty}$, to that of the radiative luminosity, $L / c$. Whereas $\mathrm{OB}$ winds are within the single-scattering limit 
$\eta \lesssim 1$ for a radiatively driven flow, inferred properties for WR winds generally imply performance numbers well in excess of this limit, typically exhibiting $\eta \simeq 10$, with extreme cases ranging to $\eta \simeq 50$ or even higher (Schmutz et al. 1989; Willis 1991; Hamann et al. 1995). Over the years, there have been many papers (Friend \& Castor 1983; Abbott \& Lucy 1986; Kato \& Iben 1992; Lucy \& Abbott 1993; Springmann 1994, 1998; Gayley, Owocki \& Cranmer 1995) examining how performance numbers above unity can in principle be obtained through multiple scattering of radiation.

Here we review the general physical requirements for achieving effective multiple scattering from a dense overlap of optically thick spectral lines, with special emphasis on the need to block the stellar flux by limiting leakage of radiation through gaps in the spectral distribution of strong wind lines. A general theme is to highlight the fundamental "opacity problem" of identifying the underlying source of the greater level of line-opacity needed to drive the much stronger mass loss of WR vs. OB stars.

\section{Multiple momentum deposition}

\subsection{Example of a static gray envelope}

The total radial momentum imparted by radiation on a spherically symmetric circumstellar envelope can be expressed in terms of the radiative force density $\rho g_{\text {rad }}$ integrated over volume, outward from the wind base at radius $r=R$,

$$
\dot{p}_{r a d}=\int_{R}^{\infty} 4 \pi r^{2} \rho g_{r a d} d r,
$$

where the radiative acceleration is given by a frequency integral of the opacity $\kappa_{\nu}$ over the stellar flux spectrum, $F_{\nu}$,

$$
g_{\text {rad }}=\int_{0}^{\infty} \frac{\kappa_{\nu} F_{\nu}}{c} d \nu
$$

A particularly simple way to illustrate the requirements of multiple momentum deposition is in terms of an envelope with a gray opacity $\kappa$ (Owocki \& Gayley 1995; Gayley et al. 1994). In this case, the flux is just a constant in frequency, set at each radius by the bolometric luminosity through $F=L / 4 \pi r^{2}$. This implies $g_{r a d}=\kappa L / 4 \pi r^{2} c$, and so yields

$$
\dot{p}_{\text {rad }}=\frac{L}{c} \int_{R}^{\infty} \kappa \rho d r=\frac{L}{c} \tau,
$$

where $\tau$ is the total wind optical depth. We thus see that the requirement for exceeding the single scattering limit for radiative momentum deposition, $\dot{p}_{\text {rad }}>L / c$, is simply that the envelope be optically thick, $\tau>1$.

Fig. 1 provides a geometric illustration of how multiple momentum deposition occurs in an optically thick envelope. Fig. 1a shows the case of a hollow shell with optical thickness $\tau=5$, wherein a photon is backscattered within the hollow sphere roughly $\tau$ times before escaping, having thus cumulatively imparted $\tau$ times the single photon momentum, as given by eq. (3). However, the same 
(a)

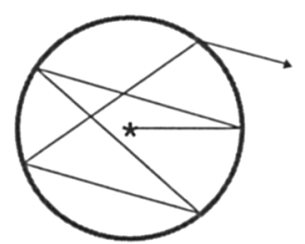

(b)

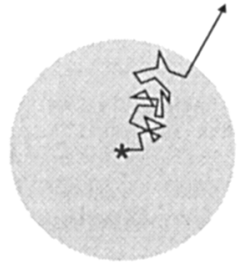

(c)

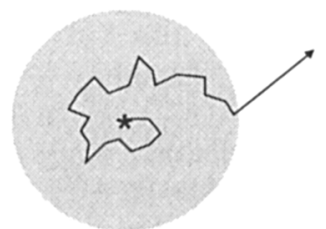

Figure 1. Multi-scattered photon trajectories in (a) hollow and (b) filled gray spheres with the same central optical $\tau=5$. The latter is shown 'untangled' in part (c) to illustrate that the multiple momentum depostion associated with hemispheric crossing in (a) is analogous to an effective 'winding' of the photon diffusion in (b).

momentum deposition also occurs in a solid sphere with the same total optical depth, even though, as shown in Fig. 1b, photons in this case undergo a much more localized diffusion without hemispheric crossing. Fig. 1c illustrates how these diffusive $v s$. direct-flight pictures of multiple momentum deposition can be reconciled by thinking in terms of an effective "winding" around the envelope. For each scattering within a spherical envelope the radial momentum deposition is unchanged by arbitrary rotations about a radius through the scattering point, including rotations that bring the scattered trajectory into a single plane, with the azimuthal component always of the same sense, say clockwise viewed from above the plane. In this artificial construction, scattering thus leads to a systematic (vs. random walk) drift of the photon along one azimuthal direction, implying a cumulative winding trajectory for which the systematic outward push of the scattered photon is now apparent.

\subsection{Multi-line transfer in an expanding wind}

The multiple scattering by a dense ensemble of lines can actually be related to the above gray envelope case, if the spectral distribution of lines is Poisson, and so is spread throughout the spectrum without extended bunches or gaps. As first noted by Friend \& Castor (1983), and later expanded on by Gayley et al. (1994), a wind driven by such an effectively gray line-distribution can be analyzed through an extension of the standard Castor, Abbott \& Klein (1975, hereafter CAK) formalism traditionally applied to the more moderate winds of OB stars. In the case of dense overlap, wherein optically thick lines in the wind have a frequency separation characterized by a velocity $\Delta v$ much less than the wind terminal speed $v_{\infty}$, the mean-free-path between photon interactions with separate lines is given by

$$
\frac{1}{\rho \kappa^{e f f}}=\frac{\Delta v}{d v_{l} / d l},
$$

where $d v_{l} / d l$ is the projected wind velocity gradient along a given direction of the photon pathlength $l$. The directional dependence of this velocity gradient implies an inherent anisotropy to the associated effectively gray line-ensemble opacity $\kappa^{e f f}$. However, a full non-isotropic diffusion analysis (Gayley et al. 1994) indicates that the overall wind dynamics is not too sensitive to this anisotropy. 
In particular, the total wind momentum can still be characterized by the effective radial optical depth, $\tau_{r}^{\text {eff }}$, which in this case yields,

$$
\dot{p}_{r a d} \simeq \frac{L}{c} \tau_{r}^{e f f}=\frac{L}{c} \int_{R}^{\infty} \rho \kappa_{r}^{e f f} d r=\frac{L}{c} \int_{R}^{\infty} \frac{1}{\Delta v} \frac{d v_{r}}{d r} d r=\frac{L}{c} \frac{v_{\infty}}{\Delta v},
$$

from which we identify $\tau_{r}^{e f f}=v_{\infty} / \Delta v$. Neglecting a modest correction for gravitational escape, global momentum balance requires $\dot{p}_{\text {rad }} \simeq \dot{M} v_{\infty}$, thus implying

$$
\eta \simeq \frac{v_{\infty}}{\Delta v}
$$

For winds driven by a gray ensemble of lines, we thus see that large performance numbers simply require that there be a large number of optically thick lines overlapping within the wind.

Note that eq. (6) implies that the mass loss scales as

$$
\dot{M} \simeq \frac{L}{c^{2}} \frac{c}{\Delta v},
$$

wherein we note that $c / \Delta v$ just represents the total, spectrum-integrated number of thick lines. It is important to realize, however, that this number of thick lines is not fixed a priori, but is itself dependent on wind properties like the mass loss rate and velocity law. Within the CAK formalism in which the line number distribution in opacity is given by a power law, one again finds that the mass loss follows a standard CAK scaling relation,

$$
\dot{M} \simeq \frac{L}{c^{2}}\left(\frac{Q \Gamma}{1-\Gamma}\right)^{(1-\alpha) / \alpha},
$$

where $\Gamma\left(\equiv \kappa_{e} L / 4 \pi G M c\right)$ is the Eddington parameter, $\alpha$ is the usual CAK exponent, and the line-opacity normalization constant $Q$ is related to the CAK constant $k$ by $k \sim Q^{1-\alpha}$ (Gayley 1995). Note that the second factor in eq. (8) simply represents the total number of thick lines, $c / \Delta v$.

As emphasized by Kudritzki et al. (1995), the CAK mass-loss law together with the tendency for the terminal speed $v_{\infty}$ to scale with the effective escape speed $v_{\text {esc }}=\sqrt{2 G M(1-\Gamma) / R}$ implies a wind-momentum-luminosity relation,

$$
\dot{M} v_{\infty} \sqrt{R} \sim L^{1 / \alpha} Q^{1 / \alpha-1}
$$

wherein we have neglected a residual dependence on $M(1-\Gamma)$ that is generally very weak for the usual case that $\alpha$ is near $2 / 3$. Fits for galactic OB supergiants (Puls et al. 1996) give a luminosity slope consistent with $\alpha \simeq 0.57$, with a normalization implying $Q \simeq 10^{3}$.

For WR stars, such comparisons of wind momentum vs. luminosity show a much greater scatter, but with momenta consistently above those inferred for OB stars, typically reflecting more than a factor 10 higher mass loss rate for the same luminosity (Hamann et al. 1995). If we assume the same $\alpha \simeq 0.6$ that characterizes $\mathrm{OB}$ winds, eq. (9) suggests that $\mathrm{WR}$ winds must have a line opacity normalization $Q$ that is more than a factor $\simeq 10^{\alpha /(1-\alpha)} \simeq 30$ higher! 
But this enhanced mass loss could also be obtained through a slightly lower $\alpha$, representing a somewhat flatter number distribution in line opacity. For example, for stars with Eddington parameter $\Gamma \simeq 1 / 2$ and O-star value for $Q \simeq 10^{3}$, simply decreasing from $\alpha=0.6$ to $\alpha=0.5$ yields the required factor $Q^{1 / 0.5-1 / 0.6} \simeq 10$ increase in $\dot{M}$.

While such a modest reduction in $\alpha$ may seem more plausible than a large increase in $Q$, it is generally not clear what basic properties of WR winds could lead to either type of change in the line opacity distribution. Viewed in this way, the fundamental distinguishing characteristic of WR winds is not so much their large performance number, but rather their enhanced mass loss compared to $\mathrm{OB}$ stars with similar luminosity. Identifying the sources of the enhanced opacity required to drive this enhanced mass loss thus represents a fundamental, unsolved "opacity problem".

\section{Momentum deposition for a non-gray line distribution}

\subsection{Monte-Carlo and NLTE models with detailed line lists}

Another essential complication for developing realistic models of WR winds stems from the inherently non-Poisson character of the spectral line distributions derived from atomic data bases of line lists (Abbott \& Lucy 1985; Lucy \& Abbott 1993). As recently elaborated by Springmann (1998), at any given wind radius, the dominant contribution to the line opacity stems from a surprisingly small number of specific ionization stages of abundant heavy metals, primarly iron and iron-group elements. Moreover, for any given ion stage, the term structure is such that the lines are notably "bunched" into relatively restricted ranges of wavelength. With just a small number of distinct ion stages, the cumulative line spectrum thus likewise exhibits extensive wavelength bunching. Within the gaps between these bunches, the radiation can propagate relatively unimpeded by line-scattering, thus representing a potential preferential "leakage" that can significantly reduce the global radiative momentum deposition.
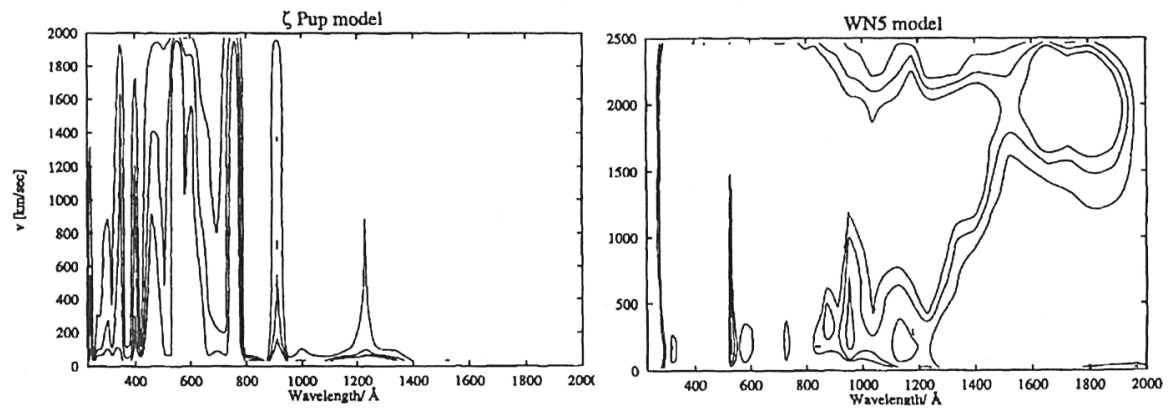

Figure 2. Contours of the number of thick wind lines as a function of wavelength and wind velocity, for both the canonical OB star $\zeta$ Pup, and a model WN5 star. From Springmann \& Puls (1997). 
A good illustration of these bunches and gaps in the line spectral distribution is given by Fig. 2, taken from Springmann \& Puls (1997). This shows contours of the number of thick wind lines as a function of wavelength and wind velocity, for both the canonical OB star $\zeta$ Pup, and a model WN5 star. Because the ionization of an $\mathrm{OB}$ wind is nearly constant, the line bunches and gaps in the $\zeta$ Pup case remain nearly fixed throughout the entire wind velocity range. By contrast, for the WR wind there is significant ionization stratification, and this leads to a mixing of the spectral positions of local bunches and gaps.

This characteristic ionization stratification of WR winds has been long known empirically through the observed inverse correlation between emissionline width and ionization potential (Beals 1941; Schulte-Ladbeck et al. 1995). But its potential significance for wind dynamics was first emphasized by Lucy \& Abbott (1993), who pointed out that the associated tendency to fill in linedistribution gaps can substantially enhance the effective global blocking of radiative flux by multi-line scattering. Using detailed line-lists compiled from an atomic database, Lucy\& Abbott carried out Monte-Carlo calculations of the transport of stellar core radiation through a WR wind with a fixed ' $\beta=1$ ' velocity law (i.e., $v(r) \simeq(1-R / \mathrm{r})^{\beta}$, with $\beta=1$ ). After iterating to obtain the globally supported mass loss rate, they found that, for the specific WN5 star model considered, the radiative momentum deposition was sufficient to balance the global momentum requirements at a performance number of $\eta \simeq 10$. However, they did not attempt to iterate to a full, local dynamical consistency, ending with a radiative acceleration that was too weak in the inner wind, and too strong in the outer wind, in both cases by up to about a factor two.

In these Monte Carlo models, a crucial simplication was to impose the ionization stratification semi-empirically by invoking an ionization radiation temperature whose inverse was taken to vary linearly with wind speed. From more fundamental non-LTE calculations, Schmutz (1997) has pointed out the crucial role of He III recombination in controlling, through the He II Lyman continuum flux, the overall ionization. To obtain the He recombination needed to induce ionization stratification, he finds it necessary to introduce a "photon loss" in the He II Ly- $\alpha$ transition. In this case, he finds models with WR mass loss rates do have sufficient radiative force to support the acceleration of the outer wind, but still lack sufficient driving in the inner regions (i.e., for $v<v_{e s c}$ ).

Recent $\mathrm{PhD}$ thesis research by U. Springmann has applied the Monte Carlo approach to investigate multi-line scattering both for the idealized, Poissondistributed, effectively gray line-distribution case (Springmann 1994), as well as for more realistic models based on a detailed line list (Springmann 1998). The former represents an approach that nicely complements, in both a computational and conceptual sense, the semi-analytic, non-isotropic diffusion analysis by Gayley et al. (1994), with good overall agreement. The two methods, for example, independently led to the conclusion that multi-line scattering should cause a somewhat more extended outer wind acceleration, a result that at least trends in the right direction to explain the quite large " $\beta$ " velocity exponents inferred by observations (e.g., Robert 1991; Lépine 1998).

Springmann's more recent calculations using a detailed line list are likewise in good general agreement with the previous results of Lucy \& Abbott (1993), but are applied to an extensive grid of WR model parameters. The 
results show increased performance numbers for stars toward the upper left of the HR diagram, with a maximum of $\eta=10.1$ for a star with $T_{\text {eff }}=90 \mathrm{kK}$ and $\log \left(L / \mathrm{L}_{\odot}\right)=5.6$. This represents a distinct enhancement (by a factor $\sim 7$ ) over the performance numbers corresponding to the empirical wind-momentumluminosity relation derived for OB supergiants. However, comparison with performance numbers from observationally inferred data tabulated by Hamann et al. (1995) shows that the theoretical models are still systematically lower, typically by a factor 10 . Some, but probably not all, of this remaining discrepancy may reflect overestimation of mass loss rates (e.g., by not properly accounting for wind clumping; Hillier 1991), or underestimation of the wind luminosity (e.g., by not properly accounting for line-blocking; Hillier \& Miller 1998).

Overall it seems optically thick WR wind models with ionization stratification provide some, but not all, of the solution to both the WR momentum and opacity problems. Moreover, even within the enhanced global wind momentum supported in such models, there is locally insufficient radiative driving in the lower regions where the wind is initiated.

\subsection{Role of line bunches, gaps, and core thermalization}

To provide a physical perspective to the above Monte Carlo and NLTE models, it is helpful to return to the concept from $\S 2.2$ that the line-ensemble constitutes an effective continuum opacity, but now allowing this to be frequency dependent to account for the relative bunches and gaps in the spectral distribution of lines. As seen from eq. (2), the radiative acceleration for such a non-gray opacity depends on the spectral integral of the opacity times the frequency-dependent radiation flux. In general the flux spectrum at any given location in an atmosphere or wind depends on self-consistent solution of a global radiation transport problem, with overall characteristics depending critically on the thermalization and frequency redistribution properties of the medium.

Figures $3 a-d$ illustrate this key role of photon thermalization and redistribution for simple non-gray line-distributions that are divided locally into two distinct spectral regions, representing either a line "bunch" or "gap". Fig. 3a first recaps the effectively gray case in which the entire spectrum is covered by lines at fixed velocity separation $\Delta v=v_{\infty} / 10$. Since this represents a total effective optical depth $\tau=10$ for photons to escape from the surface $(v=0)$ to infinity $\left(v=v_{\infty}\right)$, the global radiative momentum is $\dot{p}_{\text {rad }}=10 L / c$, as follows from eq. (3).

In Fig. $3 \mathrm{~b}$ lines have the same concentration $\Delta v=v_{\infty} / 10$ in half the spectrum (the bunch), with the other half completely line-free (the gap). Photons blocked in the bunched region are then rethermalized in the stellar core, and so tend to escape though the gap. Simple statistical arguments show that only about $0.5 /(1+\tau / 2)=1 / 12$ of the total flux now makes it out through the half of the spectrum covered by the bunch, implying from eq. (3) that the total radiative momentum is now only $\dot{p}_{\text {rad }} /(L / c) \simeq 10 \times 1 / 12+0 \times 11 / 12=5 / 6<1$ ! This roughly represents the circumstance applicable to OB star winds, which have radially constant ionization, and so a radially fixed line-spectrum. For such winds, we can see that any significant spectral gaps keep the performance number to near the single scattering limit $\dot{p}_{\text {rad }} \simeq L / c$, even if there is very extensive line overlap in spectral line bunches. 

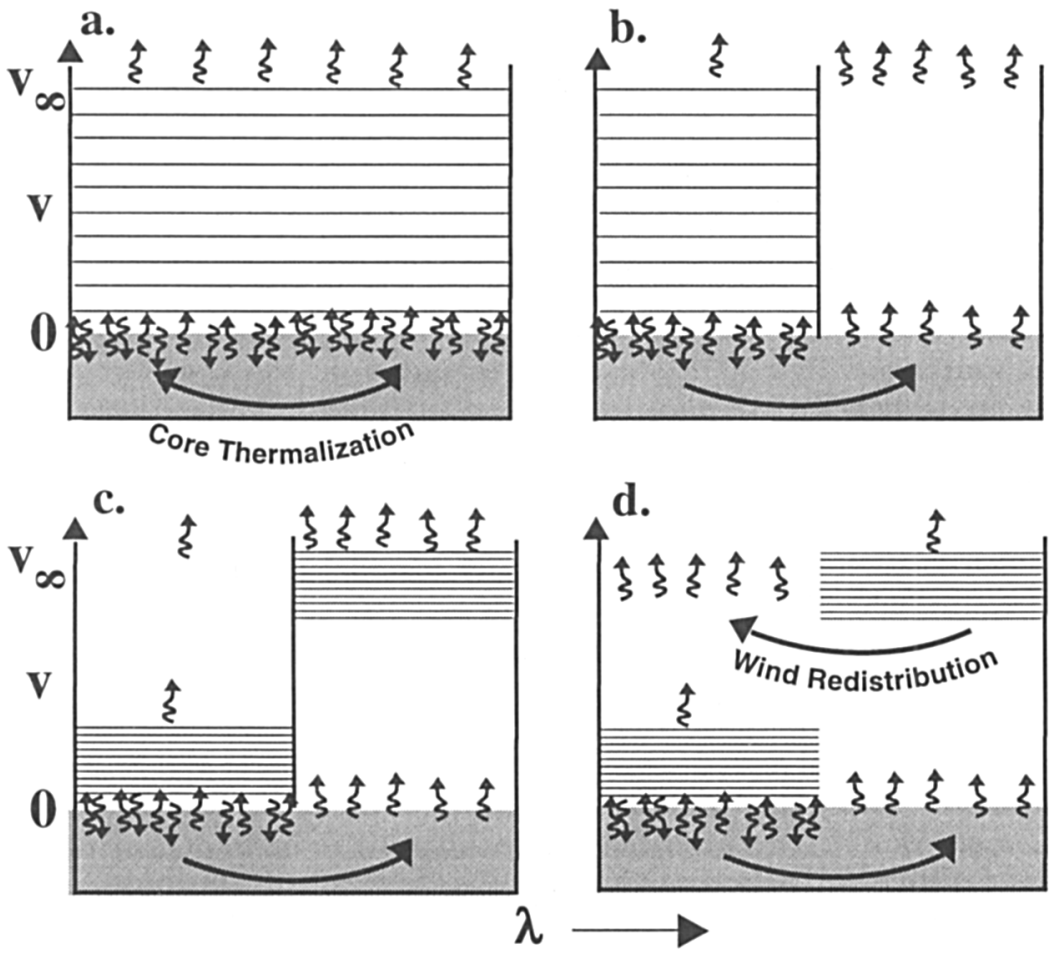

Figure 3. Schematic diagram illustrating the role of line gaps, line bunches, and photon thermalization for wind momentum deposition. As detailed in the text, the four parts represent: (a) the effectively gray model; (b) an OB wind with fixed ionization and extensive gaps; (c) a WR wind with ionization stratification that fills gaps; and (d) the importance of limiting spectral redistribution in the wind.

Fig. $3 \mathrm{c}$ represents the case wherein there are again $\tau=10$ lines covering the full spectrum, but now over a limited spatial range, occuring very close to the star in one spectral region, and very far away in the other. In the region where line-blocking occurs near the star, core thermalization again channels photons to the other spectral region. But in this second spectral region, the blocking occurs far away from the star, with greatly reduced probability of backscattering to thermal redistribution in the stellar core. Again assuming purely coherent line-scattering in the wind, this means the flux is nearly independent of the outer wind blocking. Since all the stellar flux must diffuse through a layer somewhere with $\tau=10$ lines, the global momentum deposition is now again, as in case a, simply $\dot{p}_{\text {rad }}=10 L / c$. However, note that the flux distribution is still similar to case $b$, i.e., in proportion $1 / 12$ and $11 / 12$ to the spectral regions corresponding respectively to the inner and outer wind blocking. This thus implies the same ratio for the relative deposition of radiative momentum. Overall we see that this example, intended to represent the case of an optically thick WR wind with ionization stratification, does indeed illustrate how it is possible to get a large global momentum deposition even when the line-opacity-spectrum is 
locally divided into gaps and bunches. However, it also helps explain why, as found in the detailed models described in $\S 3.1$, this radiative momentum tends to be deposited more in the outer wind, leaving a net deficit in driving needed to initiate the outflow in the lower wind.

The final example in Fig. 3d illustrates the crucial importance of the assumption that the radiative transfer within the wind itself is through pure, coherent scattering. If there is significant thermalization or any other type of spectral energy redistribution within the wind itself, then radiation will always tend to be channeled into local gaps, thus again limiting the momentum deposition to a level roughly characterized by the single scattering limit.

\subsection{Wind initiation and variability}

Detailed monitoring of WR wind emission lines provides clear evidence for an extensive, stochastic, small-scale variability (Robert 1991; Lépine 1998). Such variability might develop from the known intrinsic instability of line-driving (Owocki 1994), but the limited analysis done so far (for the effectively gray case, see Gayley et al. 1996) indicates that the growth rate of this instability should be significantly reduced, by roughly a factor of the performance number, relative to the growth in $\mathrm{OB}$ winds. An alternative is thus that the wind variability is induced by the underlying star, e.g., by pulsations. Given the particular difficulties noted above in getting a sufficiently strong radiative force in the inner wind, it is even possible to consider a "two-stage" driving process, by which strong stellar pulsations actually initiate the mass loss (Glatzel et al. 1993), with radiative forces taking over to drive the extended acceleration and high terminal speeds. Indeed, for the slow, massive winds of cool AGB stars, a quite analogous two-stage process is the standard model, with pulsations lifting and extending material to a height where dust formation allows continued flow driving by radiation (Willson 1987; Netzer \& Elitzur 1993).

Finally, even if radiative driving is by itself sufficient to initiate a WR wind, it seems plausible that the base outflow could be significantly more variable than in the O-star case. For O-stars the near radial-constancy of the ionization, and hence of the line-driving parameters, means there is a natural "compatibility" between the mass flux initiated in the inner wind, and the line-force available to drive material far from the star. In WR winds, the ionization stratification and the complexity of the photon blocking process (dependence on gaps, thermalization, etc.) means that such inner/outer wind compatibility is no longer guaranteed. In principle, this complexity might well allow for new kinds of global instabilities that would result in variable mass loss over a broad range of scales.

\section{Conclusion}

Much of the historical emphasis on a "momentum problem" for WR winds seems rooted in the intuitive notion that the momentum of a radiatively driven wind should be limited by the momentum of the radiation. In a fundamental sense, this notion is false, since we see that multiple scattering can, in principle, readily lead to multiple momentum deposition. In practice, however, the level of such multiple scattering is indeed limited by leakage through gaps in the spectral distribution of opacity, especially in the more thermalized lower regions where 
the wind is initiated. In this context, it remains relevant to cite the large performance numbers of WR winds. But it seems the key questions are why WR stars have such a larger mass loss than OB stars of comparable luminosity, and what could cause the enhanced opacity needed to drive this enhanced mass loss.

Acknowledgments. This work was supported in part by NASA grant NAGW-2624. We thank U. Springmann, J. Puls, J. Hillier, W. Hamann, and W. Schmutz for stimulating discussions.

\section{References}

Abbott, D.C., Lucy, L.B. 1985, ApJ 679, 693

Beals, C.S. 1941, in: A. Shaler (ed.), Observation des Novae (Paris: Hermann)

Castor, J.I., Abbott, D.C., Klein, R.I. 1975, ApJ 195, 157 (CAK)

Friend, D.B., Castor, J.I. 1983, ApJ 272, 259

Gayley, K.G. 1995, ApJ 454, 410

Gayley, K.G., Owocki, S.P., Cranmer, S.R. 1994, ApJ 442, 296

Glatzel, W., Kiriakidis, M., Fricke, K.J. 1993, MNRAS 262, 7

Hamann, W.-R., Koesterke, L., Wessolowski, U. 1995, A\&A 299, 151

Hillier, D.J. 1991, A\&A 247, 455

Hillier, D.J., Millier, D.L. 1998, ApJ 496, 407

Kato, M., Iben, I. 1992, ApJ 394, 305

Kudritzki, R.-P., Lennon, D., Puls, J. 1995, in: J. Walsh \& I. Danziger (eds.), Science with the Very Large Telescope, (ESO: Garching)

Netzer, N., Elitzur, M. 1993, ApJ 410, 701

Lépine, S. 1998, PhD Thesis, Université de Montréal

Lucy, L.B., Abbott, D.C. 1993, ApJ 405, 738

Owocki, S.P. 1994, in: A.F.J. Moffat, S.P. Owocki, A.W. Fullerton \& N. St-Louis (eds.), Instability and Variability of Hot-Star Winds, ApSS 221, 3

Owocki, S.P., Gayley, K.G. 1995, in: K.A. van der Hucht and P.M. Williams (eds.), WolfRayet Stars: Binaries, Colliding Winds, Evolution, Proc. IAU Symp. No. 163 (Dordrecht: Kluwer), p. 138

Puls, J., Kudritzki, R.-P., Herrero, A. et al. 1996, A\&A 305, 171

Robert, C. 1991, PhD Thesis, Université de Montréal

Schmutz, W., Hamann, W.-R., Wessolowski, U. 1989, A\&A 210, 236

Schmutz, W. 1997, A\&A 321, 268

Schulte-Ladbeck, R.E., Eenens, P., Davis, K. 1995, ApJ 454, 917

Springmann, U. 1994, A\&A 289, 505

Springmann, U., Puls, J. 1997, in: I. Howarth (ed.), Boulder-Munich II: Properties of Hot, Luminous Stars, ASP-CS 131, 286

Springmann, U. 1998, Ph.D. Thesis, University of Munich

Willis, A. J. 1991, in: K.A van der Hucht \& B. Hidayat (eds.), Wolf-Rayet Stars and Interrelations with Other Massive Stars in Galaxies, Proc. IAU Symp. No. 143, (Dordrecht: Kluwer), p. 265

Willson, L. 1987, in: Late Stages of Stellar Evolution (Dordrecht: Reidel), p. 253 


\section{Discussion}

Leitherer: What is the physical reason for the question 'Why is $\dot{M}_{\mathrm{WR}} \simeq 10 \times \dot{M}_{\mathrm{O}}$ ?' LBVs are the evolutionary progenitors of WR stars. In principle there would be a momentum problem for LBVs as well, were it not for a lower $v_{\infty}$. Could one re-state the problem as 'Why is $v_{\infty}(\mathrm{WR}) \simeq 10 \times v_{\infty}(\mathrm{LBV})$ ?'

Owocki: Quiescent phase LBVs and Of/WN stars appear to represent a transition for whatever process is making $\dot{M}$ increase from $\dot{M}_{\mathrm{O}}$ to $\dot{M}_{\mathrm{WR}}$; but in these stars the density may not yet be high enough to make the wind optically thick, with the associated ionization gradients. As such, they would not experience this 'afterburner' effect of extended, outer-wind acceleration. Another factor is the much larger $R_{x}$ in LBVs and Of/WN vs. WR stars. This implies a lower surface escape speed, and within standard line-driven wind theory a lower $v_{\infty}$. Finally, the $\sqrt{R}$ correction to the wind-momentumluminosity relation would also imply a lower $\dot{M} v_{\infty}$ for LBVs vs. WRs, even apart from the overall tendency to higher effective opacity for driving WR mass loss.

Schmutz: Sergey Marchenko showed in his talk that there are two winds of WR stars: those that have already a large expansion velocity at the photosphere, and those that have transparent winds down to basically zero velocity. Thus, two different acceleration mechanisms must operate. For the first type the creation of the wind occurs in the optically thick part of the wind and for the second type, the whole acceleration is in the optically thin part. The latter must be line-driven like the winds of $\mathrm{O}$ stars but yet, their winds are much slower for given luminosity; whatever the reason.

Owocki: I see these as not qualitatively distinct, but rather a question of degree in wind optical thickness to electron scattering, which, in my view, should not alter the basic requirements for line-driving to be effective. Optical thickness to electron scattering will change the mean intensity and so makes the radiation field appear more isotropic, but the flux, which is the key for radiative driving, will not be affected.

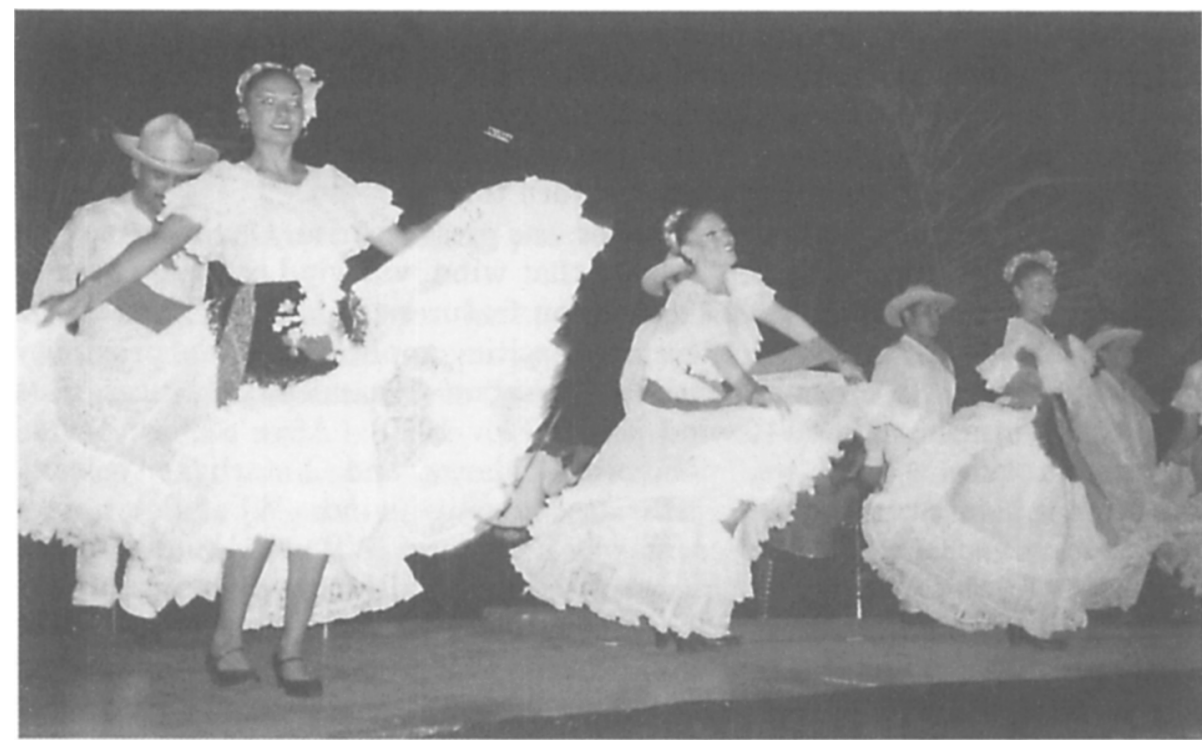

